\title{
TOXICOLOGÍA AMBIENTAL EN MINERÍA: Herramientas para la evaluación del impacto
}

\author{
Darío Zegarra Macchiavello \\ M.SC. EN CIENCIAS AMBIENTALES, UNIVERSITY OF IDAHO \\ Óscar Frías Martinelli \\ PROFESOR CONTRATADO DE ESAN \\ Ph.D. (C) En EConomía DE Minerales, Penn State University
}

\begin{abstract}
Resumen
El creciente interés por los efectos que la minería causa en el ambiente ha dado origen a nuevas formas de prevención y control en esta industria. La puesta en marcha de una operación minera puede entenderse como una alteración de un ecosistema en equilibrio, es ya parte de un sistema buscando equilibrio. Pero, ¿qué tanto ha sido roto el equilibrio y por cuánto tiempo?, ¿cómo cuantificar el efecto sobre los inventarios de biota del área afectada?, ¿cómo tomar en cuenta la bioacumulación de los xenobioticos en el hábitat?, ¿cómo incluir tantas variables, sus relaciones y su evolución temporal? El análisis a través del tiempo de estas alteraciones o redefiniciones de las variables presentes en el ambiente debe ser tomado en cuenta para un estudio del impacto en el ambiente. Este trabajo presenta criterios y herramientas para el análisis de los efectos que una operación minera o industrial causa en su medio circundante. Se analizan la Evaluación Probabilística de Riesgos (o Simulación Monte Carlo) y el Modelamiento de Sistemas Dinámicos, herramientas cuya base común es la simulación de modelos como enfoque para el desarrollo de representaciones resumidas de la realidad. A fin de mostrar la practicidad, relevancia y aplicabilidad de estos enfoques, se revisa un caso de estudio de cada uno de ellos.
\end{abstract}

\section{Introducción}

La toxicología ambiental es la ciencia que estudia las formas y medios por los cuales los agentes contaminantes alteran el equilibrio biológico de las poblaciones, comunidades y hábitat. Es una disciplina que, basada en el análisis de las interrelaciones físicas y químicas de las variables que componen un determinado hábitat (aire, agua, suelo, biota), busca definir los posibles efectos que la minería u otra actividad causan en la salud de los organismos vivos.

La evaluación del riesgo de contaminación ambiental incluye muestreos de campo de ambientes terrestres y acuáti- 
cos. Asimismo, la evaluación de riesgo estudia el transporte, bioacumulación, almacenamiento, toxicidad y caracterización del riesgo. La evaluación del riesgo para la salud humana incluye, además, modelos predictivos de la amenaza a la salud por agentes ambientales a los que determinada población esta expuesta. Cuando esta exposición es sistemática, la amenaza es expresada en términos de un coeficiente de riesgo, que por lo general es aceptable si es menor de la unidad. Un concepto asociado al cálculo del riesgo ambiental relacionado con el potencial efecto en la salud humana es la Dosis, cuya definición incluye tanto la emisión puntual como la exposición a todas las demás fuentes que aportan el xenobiotico al ambiente. La dosis se calcula generalmente con la siguiente fórmula:

Dosis $=(\mathrm{CA} * \mathrm{RC} * \mathrm{FE} * \mathrm{PC}) /(\mathrm{PC} * \mathrm{FC})$

Donde:

$\mathrm{CA}=$ Concentración del agente contaminante en el medio de potencial exposición ( $\mathrm{mg} / \mathrm{l}$ )

$\mathrm{RC}=$ Ratio de contacto con el medio de potencial riesgo (1/d)

$\mathrm{FE}=$ Frecuencia de exposición (d/a)

$\mathrm{PC}=$ Peso corporal $(\mathrm{kg})$

$\mathrm{FC}=$ Factor de conversión $(\mathrm{d} / \mathrm{a})$

La toxicología ambiental incluye, como uno de sus puntos más relevantes, la Evaluación de la Exposición, que considera la disponibilidad de los tóxicos para la biota.

Un aspecto resaltante es la preocupación por la determinación del uso final de la tierra y el agua luego de una restauración minera. Por ejemplo, es evidente que si el uso posterior previsto para la tierra es la agricultura, las condiciones serán completa y absolutamente distintas a si el uso posterior es el urbanismo. Las figuras 1 y 2 representan un modelo desarrollado por uno de los autores (Zegarra, 2002) en una zona minera recuperada para pastoreo. Se trata de un ejemplo de aplicación de la herramienta de Modelamiento de Sistemas Dinámicos.

Desafortunadamente, en esa ocasión las medidas técnicas aplicadas por la ingeniería de diseño de botaderos de desmonte no fueron suficientes para evitar la movilización de los óxidos de selenio al ambiente. Cientos de animales de pastoreo murieron intoxicados por contenidos elevados de selenio en las plantas. Hoy los responsables mineros enfrentan diálogos muy duros entre los rancheros y las autoridades en la zona.

\section{Evaluación probabilística de riesgo toxicológico}

La simulación Monte Carlo para la evaluación de riesgos ambientales es una idea relativamente nueva, que ha sido posible gracias a la potencia alcanzada por los computadores personales hoy disponibles. La idea fundamental es tomar un escenario donde el riesgo esté asociado a una variable, tal como aumento en los índices de cáncer cuando hay altos niveles de químicos en el ambiente. El nivel del químico es luego modelado como función de otras variables, algunas de las cuales son variables aleatorias, y la distribución de la variable de interés se genera a través de una simulación. Es entonces posible, por ejemplo, determinar la probabilidad de que la variable de interés exceda algún nivel previsto como aceptable.

El enfoque Monte Carlo comprende básicamente cinco pasos: 


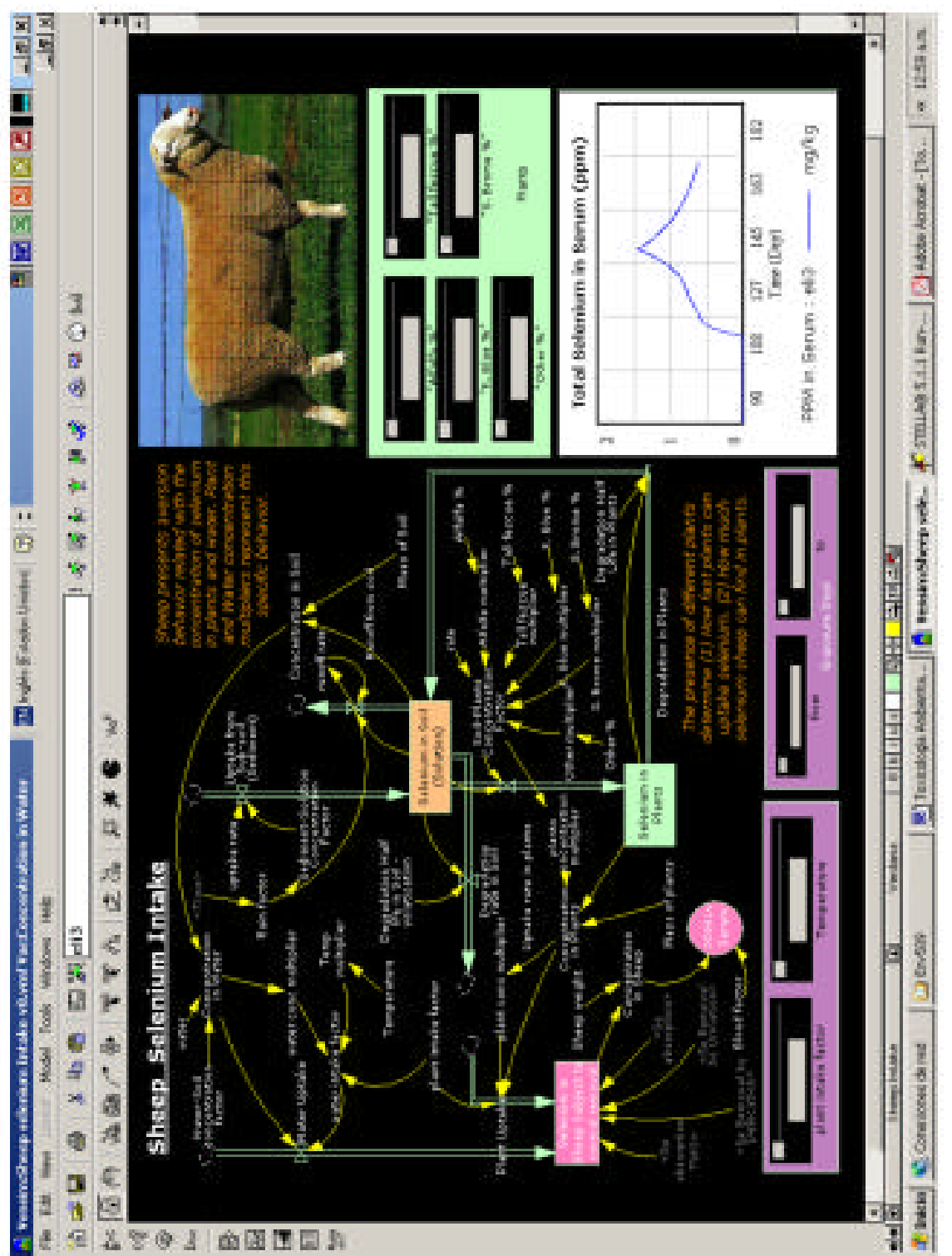

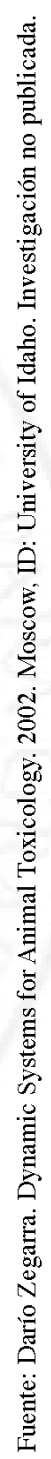



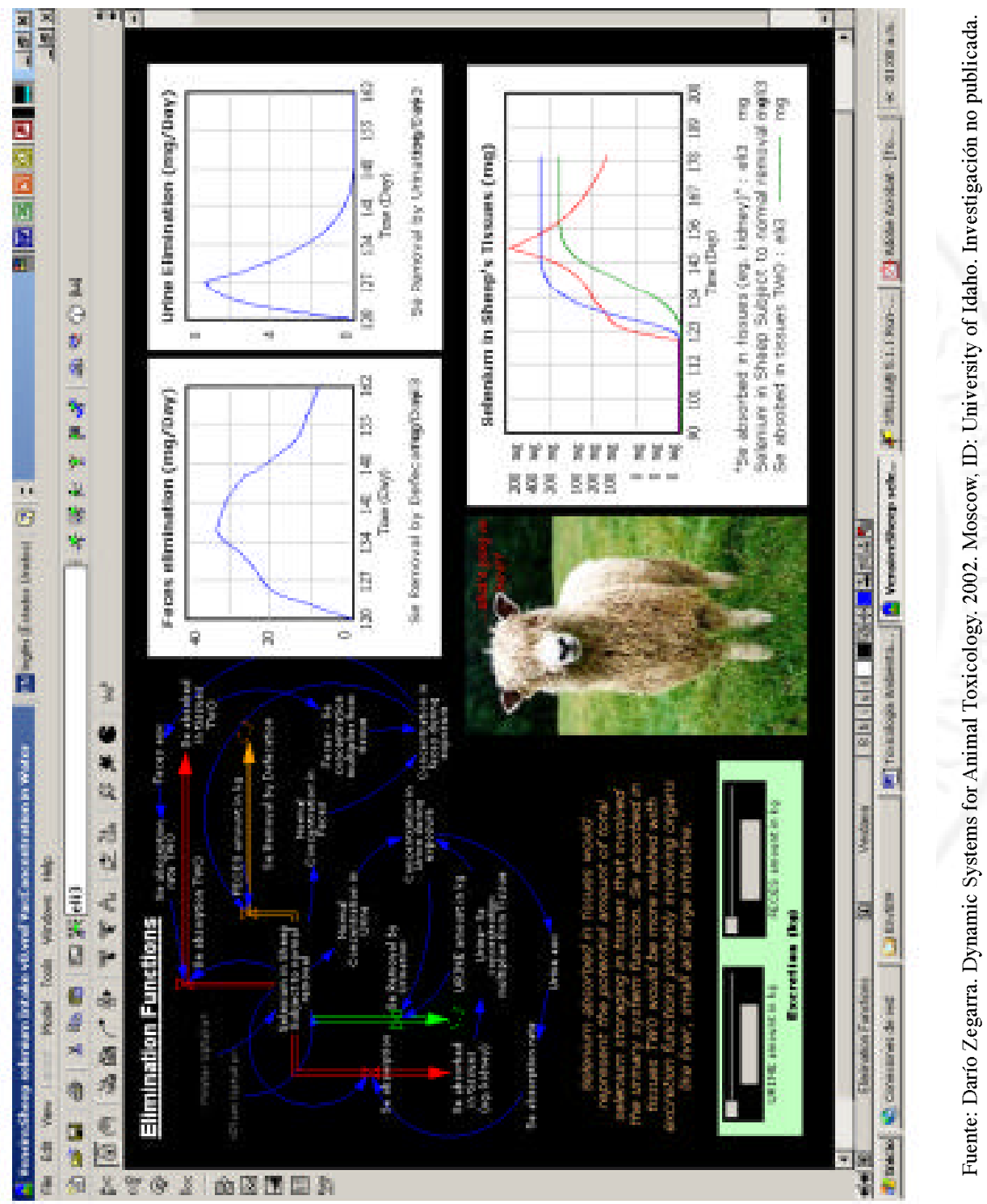
1) Se construye un modelo para describir el escenario de interés.

2) Se asumen distribuciones estadísticas para las variables aleatorias de entrada, tales como concentración química de los contaminantes en el ambiente de estudio, frecuencias de exposición, etc.

3) Se definen los resultados de interés, tales como la cantidad de exposición proveniente de diferentes fuentes, la exposición total, etc.

4) Se generan valores aleatorios para las distribuciones estadísticas de las variables de entrada, y se derivan las distribuciones de los resultados de interés.

5) Los resultados deben cumplir el estándar del análisis estadístico: media, mediana, coeficientes de variación, valor excedido el $5 \%$ de las veces, etc.

Las razones para utilizar este enfoque son: i) Los enfoques determinísticos por lo general buscan asumir el peor de los escenarios para cada variable de entrada. Esto puede originar resultados absurdos, tal como el Superfund en Oroville, California, Estados Unidos, donde se especificó un objetivo de limpieza o recuperación de 5.3E-7 ug/l para dioxinas en el agua subterránea. Curiosamente, este objetivo es 100 veces más bajo que los estándares para agua potable y 20 veces más bajo que los actuales límites de detección. De esta forma pueden existir estimados de riesgo poco razonables, lo que pone en duda todo el proceso de evaluación. ii) Manejada propiamente, una evaluación probabilística de riesgos proporciona más información que un estudio determinístico. Es posible que, en promedio, los va- lores a los que el común de la población está expuesta para determinado agente contaminante sean bajos, pero ocasionalmente habrá individuos que pueden enfrentar valores extremos. Es importante conocer esto y, en todo caso, el mundo real es estocástico más que determinístico; en otras palabras, en esencia las evaluaciones determinísticas son inherentemente insatisfactorias.

Por otro lado, es necesario rescatar que los modelos de simulación que incluyen estocasticidad son realmente necesarios sólo en las situaciones extremas, cuando el «peor de los casos» en el escenario determinístico sugiere que puede haber un problema.

\section{Modelamiento de sistemas dinámicos}

Las ideas fundamentales que dan origen a la disciplina de los Sistemas Dinámicos son desarrolladas por Jay Forrester en la Escuela de Negocios de Sloan en el Instituto Tecnológico de Massachusetts, MIT. El primer trabajo fue publicado en 1961 y estuvo enteramente dedicado a dinámicas industriales. Forrester y sus colegas desarrollaron sus ideas iniciales aplicando básicamente conceptos de retroalimentación de la Teoría de Control de sistemas industriales. A pesar de que el origen de estas técnicas está en el control, su enfoque conceptual fortalecido en las interrelaciones de los componentes de un sistema y su entendimiento holístico, basado en el comportamiento de variables de control en diferentes escenarios, ha permitido la extrapolación de las técnicas a disciplinas tan variadas como la dinámica de poblaciones, los mercados de capitales, la psicología y la sociología. 
El Transporte de Contaminación Atmosférica puede ser analizado también por los principios del Modelamiento de Sistemas Dinámicos. En este trabajo presentamos una alternativa de análisis para una situación de polución atmosférica en las inmediaciones de una población en riesgo.

Por lo general, las ocurrencias de contaminación atmosférica presentan una geografía muy amplia. El emisor de la contaminación es llamado fuente de polución; el área geográfica que recibe la contaminación es llamada receptor de polución. La razón por la que la contaminación atmosférica usualmente presenta esta característica fuente-receptor es porque muchos de los procesos físicos y químicos que la originan tienen lugar en un médium -la atmósfera- que es espacialmente dinámico.

Podemos referirnos a la atmósfera como el «reactor químico» donde los agentes tóxicos son transportados por el viento y factores climáticos principalmente. Las condiciones meteorológicamente dinámicas, tales como temperatura, humedad, dirección y velocidad del viento, afectan la manera como los químicos son mezclados, dispersados y transportados a través de las fronteras geográficas.

En este trabajo exploramos la naturaleza dinámica de la química atmosférica a través del desarrollo del siguiente escenario: la deposición de ácido sulfúrico formado desde la liberación industrial (refinería) de $\mathrm{SO}_{2}$. La autoridad local está preocupada por el inicio de una nueva refinería que se construirá a 60 kilómetros de distancia. Esta nueva instalación emitirá cantidades significativas de $\mathrm{SO}_{2}$. El objetivo del estudio es analizar el impac- to que estas emisiones pueden tener como deposición ácida.

Este análisis es posible con la ayuda de herramientas modernas de Simulación Dinámica. En tal sentido, se ha desarrollado una aplicación como la presentada en la figura 3.

Este modelo presenta la capacidad de acumulación y deposición de $\mathrm{SO}_{2}$ en el sistema de estudio. Fácilmente podemos entenderlo como el efecto de una refinería sobre una población. Los gráficos son totalmente dinámicos y nos permiten ensayar diferentes escenarios para las variables de interés. Se muestra la evolución en el tiempo y distancia de $\mathrm{SO}_{2}$ y $\mathrm{SO}_{3}$. $\mathrm{La}$ simplicidad del modelo mostrado se debe a fines estrictamente didácticos; es posible, por supuesto, incluir tanta complejidad como se requiera. El número de variables no tiene límite, quizás una regla práctica sea incluir sólo aquellas que aporten, en el mejor entendimiento del fenómeno, una mejor compresión del sistema y de su comportamiento.

Una de las mayores ventajas de utilizar este enfoque para la estimación de impacto es que nos permite un mejor entendimiento no sólo de las variables involucradas, sino además de las interrelaciones.

Algunas de las condiciones asumidas en el modelo desarrollado son: i) el receptor se encuentra directamente «viento abajo», ii) la dirección y velocidad del viento son constantes, iii) las emisiones de la refinería son asumidas constantes, iv) los ratios de transformación y deposición son constantes, v) el aporte natural de $\mathrm{SO}_{2}$ al sistema se asume constante y vi) se asume pulsos de emisión de la refinería. La 


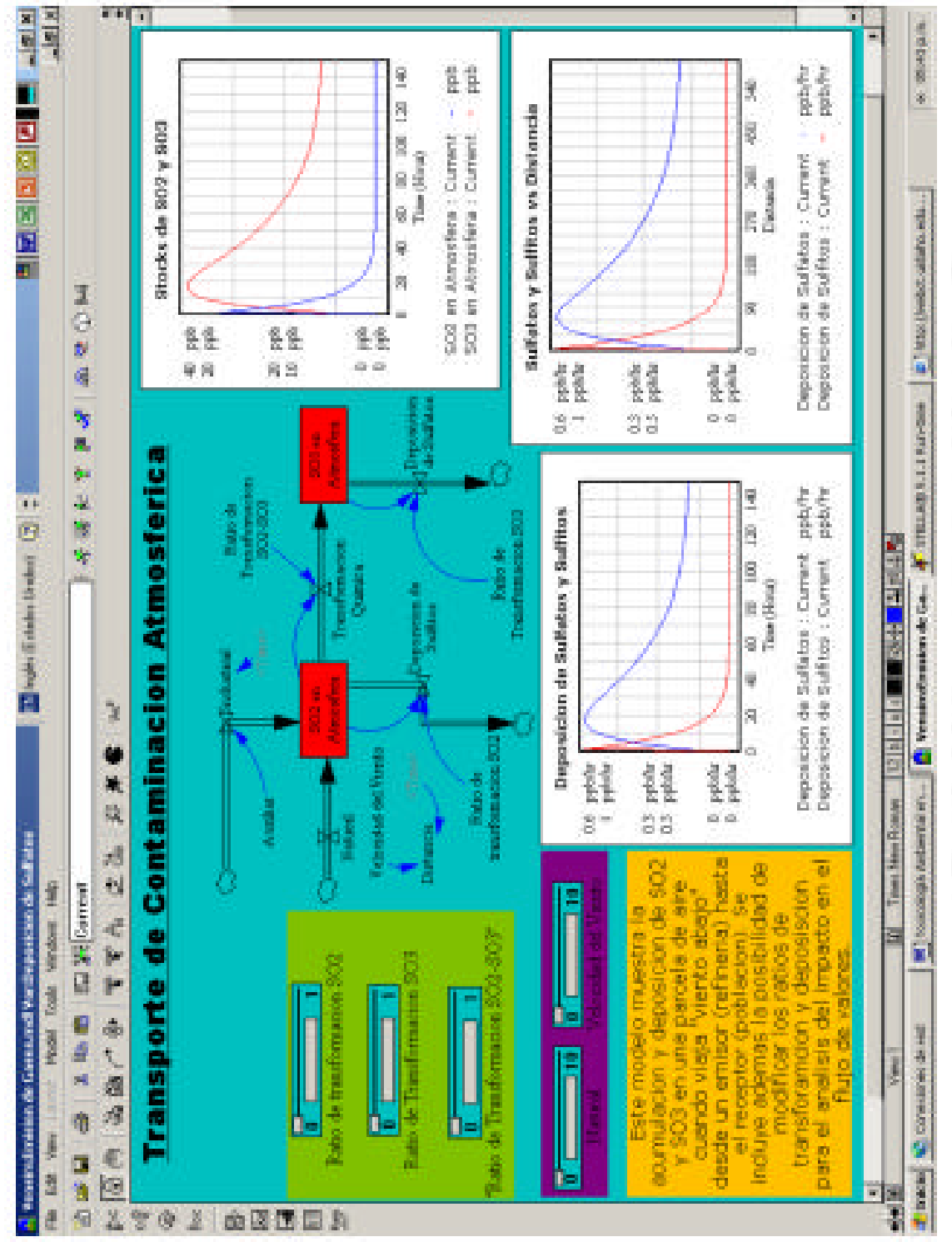

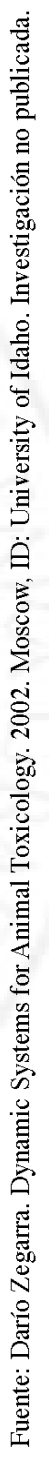

$\underset{5}{\infty}$ 
contaminación no se produce sólo una vez en el tiempo. Se definen unidades atmosféricas que reciben los tóxicos y luego viajan movilizadas por el viento. La refinería continúa contaminando sucesivamente unidades o parcelas atmosféricas.

Podemos precisar nuevamente que las simplificaciones del modelo no tienen otra justificación que la del objetivo didáctico. La inclusión de mayores complejidades demandaría un esfuerzo adicional en el modelamiento, pero no significaría mayor dificultad en su desarrollo. Explorando el modelo podemos intentar diferentes niveles de emisiones de la refinería y establecer su efecto en la deposición ácida en tiempo y distancia. Es interesante puntualizar la enorme ventaja que ofrece un modelamiento de este tipo para el entendimiento del sistema y del comportamiento de las variables de control en diversos escenarios probables.

Luego de experimentar con el modelo podemos reconocer que el sistema así concebido busca intrínsecamente el equilibrio, y el nivel de este equilibrio es independiente del pulso contaminante; sin embargo, probablemente sí dependa de la frecuencia de los pulsos, la que -podemos decirlo intuitivamente- estaría gobernada en gran medida por la velocidad del viento. En otras palabras, el análisis nos permite concluir que si consideramos eventos aislados de contaminación atmosférica suficientemente intercalados en el tiempo, la naturaleza tiene la capacidad de diluir el contaminante y alcanzar niveles no tóxicos, sin importar que tan intensa haya sido la emisión de los químicos. Sin embargo, el incremento de la frecuencia de emisión afecta significativamente la capacidad de autocontrol de la naturaleza.
Esta capacidad de autocontrol está gobernada por condiciones intrínsecas al medio ambiente en estudio, tales como variaciones de temperatura, viento, dirección del viento, intensidad de las precipitaciones y su variabilidad, presencia de poblaciones, otras industrias, etc. Los valores de estas variables y sus interrelaciones definen un ecosistema único.

El Modelamiento de Sistemas Dinámicos nos presenta la alternativa de no tratar todos los ecosistemas como «iguales». Los planes de monitoreo ambiental se centran primariamente en no exceder los Límites Máximos Permisibles (LMP) establecidos para agua, aire y suelo obligados por ley. Es decir, independientemente de cuáles sean las particularidades del ecosistema, las emisiones o efluentes de una industria no pueden exceder un límite máximo. La industria tiene la oportunidad de dedicarse no sólo a mantener sus efluentes o emisiones por debajo de estos límites legales, sino proactivamente a dedicar esfuerzos a prever los efectos de su operación en los ecosistemas circundantes.

\section{Desafío}

Si un conjunto de industrias mantiene sus emisiones de sulfuros por debajo de los LMP, ¿podemos asegurar que el ecosistema no se está alterando permanentemente?, ¿cómo identificar la sensibilidad de las variables que componen el ecosistema?, ¿qué oportunidades tenemos para impactar positivamente en el ambiente y mejorar el uso del suelo y el agua? Preguntas como éstas son factibles de responder con enfoques de modelamiento y probabilidad. 
Estamos llamados a ser responsables con nuestro ecosistema y con la población. No cabe duda de que, en gran medida, lo que nuestro país necesita es mayor oferta de empleo, y probablemente el logro de este objetivo significará mayor presencia de industrias, entre ellas operaciones mineras, fundiciones, refinerías, transporte pesado. Todo esto traerá como consecuencia una alteración inevitablemente mayor del ecosistema y, por ende, de las condiciones de vida de nuestros pueblos. Hoy existen herramientas para evaluar con mayor certeza los impactos que acarrea la tan promocionada y necesitada industrialización. La búsqueda de un equilibrio sano, de convivencia entre ecosistemas saludables y desarrollo, pasa sin duda por un mejor entendimiento de las variables en juego y de los riesgos que estamos dispuestos a afrontar.

\section{Referencias bibliográficas}

DEATON, M. y WINEBRAKE, J. 1999. Dynamic Modeling of Environmental Systems. New York: Springer.

FORD, A. 1999. Modeling the Environment. Washington D. C.: Island Press.

HANNON, B. y RUTH, M. 1994. Dynamic Modeling. New York: Springer.

KELTON, D.; SADOWSKI, R. y SADOWSKI, D. 1998. Simulation with Arena. Washington: Wiley.
MANLY, B. 2001. Statistics for Environmental Science and Management. Boca Raton: Chapman \& Hall.

MOLLER, G. 2002. Principles of Toxicology. Lecture 12 notes. Moscow, ID: University of Idaho.

ZEGARRA, D. 2002. Dynamic Systems for Animal Toxicology. Moscow, ID: University of Idaho. Investigación no publicada. 